\title{
A reflection on students' Self-Regulated Learning and the role of the academic skills advisor during Covid-19
}

\author{
Alexandra Read \\ University of Suffolk, UK
}

Keywords: academic skills; Self-Regulated Learning; virtual classroom; Covid-19.

\section{The challenge}

Our university is a newer institution embedded in the heart of the local community and focused on widening participation. The student body is made up of approximately 5,000 students, with a high percentage of first generation and mature students. My role as an Academic Skills Advisor (ASA) is to support all students in developing the skills needed to succeed in their course, and I am specifically aligned to support the academic needs within the school of Health and Sports Sciences.

In March 2020, universities across the UK were forced to close physical campuses to protect staff and students from the threat of Covid-19. This resulted in my role rapidly shifting from face-to-face (f2f) support into the online world of video calls, emails, and virtual classrooms. The biggest impact on the student/ASA relationship, was that we were now infringing on each other's private worlds; with cats walking across keyboards, children being home-schooled, or the postman at the door. Inevitably, and understandably, the biggest challenge I faced was how to effectively support stressed and emotionally drained students through virtual means.

When conducing f2f tutorials on campus, students' academic needs are often clear: assignment planning, writing structure, referencing, critical thinking, etc. However, the rapid shift in modes of learning due to the pandemic, left not only the students facing unknown learning experiences, but also posed a dilemma for myself, as I too was unaware of how to effectively support students' academic needs in this situation. However, video calls created new opportunities for me to discovery how students' academic needs had 
changed. It gave us a glimpse into each other worlds, breaking down previously unseen barriers and enabled more personal discussions. Whether this was compliments on bookshelves in the background or a child interrupting to wave hello; these conversation starters led us to have more personal and shared experiences. Students were struggling to engage with learning as they had a plethora of other worries: managing caring responsibilities, fears and sometimes grief due to Covid-19, and many with anxieties about completing placements on 'the front line' in health care roles. Covid-19 presented them with study environments that were isolating them from friends, family, and peers due to lockdowns or were chaotic with the demands of busy households, both of which left students no choice but to try to develop and adapt Self-Regulated Learning (S-RL) strategies to cope.

I received a high volume of requests for academic support from health students who were at various stages of completing their dissertations. They expressed the feeling that they had been 'left to get on with it' or did not know where to start, in addition to sharing the impact of poor emotional and mental wellbeing on their motivation to study. It appeared they were seeking practical support and advice to help motivate and manage their studies, while navigating challenges such as confinement, home schooling, caring responsibilities, and placements, some of which were on high-risk Covid-19 wards. They required a quick solution to support their S-RL while completing dissertations to ensure they did not risk developing 'suboptimal learning tactics' (Jovanović et al., 2017, p.75); students who feel under pressure are likely to quickly adopt new but incompetent S-RL strategies resulting in poor outcomes.

\section{The response}

The initial step was to decide what form of academic support could be implemented to specifically target these students' needs in completing their dissertations. This was done by drawing upon research from Jansen et al. (2019, pp.3-4) who explain that academic interventions will differ in 'cognitive activities' depending on the student's stage in higher education. Students in the early years of study require a higher level of cognitive tasks and instructional advice, whereas students in later stages require support rather than instruction (Jansen et al., 2019, p. 4). Therefore, I recognised that my role in this specific academic intervention was one of facilitating students' S-RL. 
Secondly, it was understood that establishing successful S-RL techniques is not an isolated process but rather relies on numerous intrinsic factors such as self-awareness, personal strengths, weakness, adaptability, and self-motivation (Zimmerman, 2002, pp.65$6)$. In addition, external factors highlighted by Bryan such as 'culture, class, gender, mental health and well-being' also hold a significant importance in this engagement process (2015, pp.26-7). For these reasons, it was vital to acknowledge the internal and external factors that these students were experiencing in order to provide a successful solution that would both facilitate students' motivation and help them engage effective S-RL. This was done through Maslow's (1943) hierarchy of needs (Figure 1), in which an individual must have all their needs met, at each level in order to reach self-actualisation or, in this situation, successfully complete their dissertations.

Figure 1. Maslow's hierarchy of needs. (1943, in Jordan, 2008, p.156).

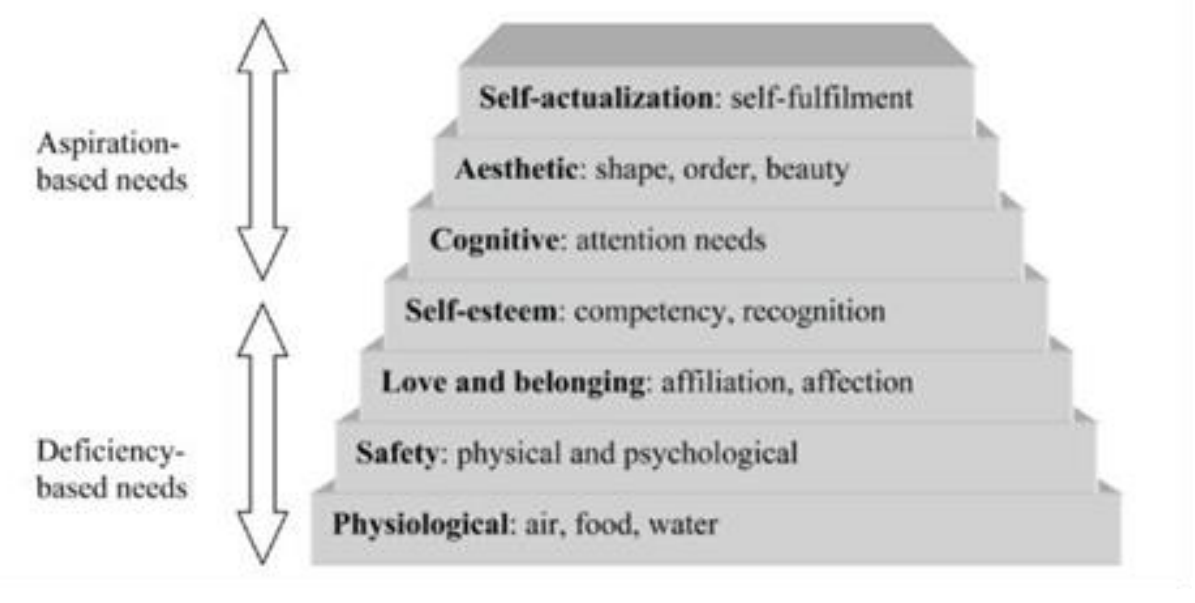

In the first instance, I applied this framework as a tool to identify and understand how, at each level, the pandemic was impacting their ability to engage $S-R L$ to complete their dissertation (self-actualisation), through lack of physical, psychological, and emotional needs. The effects of not 'having basic physiological, social and emotional needs reasonably satisfied', according to Bryan (2015, p.27) is a significant factor in students' ability or inability, to engage in learning. This process then enabled me to develop a workshop that could facilitate support at each level, to ensure the students could complete their dissertations. The 'Write here, Write now' workshop was delivered via virtual classroom, for two hours at various times. Prior to the session, students received a presession plan to create their own aims/targets. The session itself was structured around a 
time management technique of 25 minutes self-directed work, with 10-15 minute interval breaks to engage with myself and peers, ending the session with reflection and next steps.

\section{Recommendations}

Through applying both Jansen et al.'s (2019) research and Maslow's (1943) hierarchy of needs, I was able to plan and consider how 'Write here, Write now' could provide the solution the students needed to successfully engage their S-RL. Breaking this down specifically regarding each level:

- Physiological and safety: the pre-session plan encourages students to ensure they have refreshments to hand before the start of the workshop, and prompts students to consider how to create a productive environment.

- Love, belonging, and self-esteem: the virtual classroom advocates a safe space where students can interact with peers, fostering a sense of belonging, and receive support and encouragement.

- Cognitive and aesthetic: the workshop is designed to facilitate students to engage pre-existing S-RL and models how to implement these skills independently, alongside the opportunity to participate in activities to help stimulate creativity.

Ultimately, the response of facilitating a workshop to target a group of students who were struggling emotionally, mentally, and physically to engage $\mathrm{S}-\mathrm{RL}$ resulted in the successful completion of dissertations in these unprecedented times.

The recommendations are based on the consideration given to the wider factors that can impact students' ability to engage in S-RL and how applying a holistic framework such as Maslow's can be a tool to embed this approach into workshop design.

\section{References}

Bryan, C. (2015) 'Enhancing student learning', in Lea, J. (ed.) Enhancing learning and teaching in higher education. engaging with the dimensions of practice.

Maidenhead: Open University Press, pp.20-53. 
Jansen, R. S., van Leeuwen, A., Janssen, J., Jak, S. and Kester, L. (2019) 'Self-regulated learning partially mediates the effect of self-regulated learning interventions on achievement in higher education: a meta-analysis', Educational Research Review, 28, pp.1-20. https://doi.org/10.1016/j.edurev.2019.100292.

Jordan, A. (2008) Approaches to learning. Maidenhead: Open University Press.

Jovanović, J., Gǎsević, D., Dawson, S., Pardo, A. and Mirriahi, N. (2017) 'Learning analytics to unveil learning strategies in a flipped classroom', Internet and Higher Education, 33, pp.74-85. Available at: https://doi.org/10.1016/j.iheduc.2017.02.001.

Zimmerman, B. J. (2002) 'Becoming a self-regulated learner: an overview', Theory into Practice, 41(2), pp. 64-70. https://doi.org/10.1207/s15430421tip4102 2.

\section{Author details}

Alexandra Read is an Academic Skills Advisor within the Department of Library and Learning Services at the University of Suffolk, with knowledge and experience of supporting children, young people, and adult learners with SENDS and SpLDs. She has a particular interest in developing an inclusive HE practice that supports all students from diverse and widening participation backgrounds. In addition, Alexandra is keen to continue with her professional development through reflective practice, evidenced based research projects, and collaborations with professionals across a variety of HEls. 